\title{
Three-Dimensional Scenes Restore Using Digital Image
}

\author{
Takialddin Al Smadi' ${ }^{1}$ Igried Al-Khawaldeh ${ }^{1,2}$, Kalid Al Smadi ${ }^{3}$ \\ ${ }^{1}$ Department of Communications and Electronics Engineering, College of Engineering, Jerash University, Jerash, Jordan \\ ${ }^{2}$ College of Engineering Technology, Al-Balqa Applied University, Salt, Jordan \\ ${ }^{3}$ Jordanian Sudanese Colleges for Science \& Technology, Khartoum, Sudan \\ Email: Takialddina@yahoo.com, igriedgm@gmail.com
}

How to cite this paper: Al Smadi, T., AlKhawaldeh, I. and Al Smadi, K. (2017) ThreeDimensional Scenes Restore Using Digital Image. Journal of Signal and Information Processing, 8, 1-8.

https://doi.org/10.4236/jsip.2017.81001

Received: March 30, 2016

Accepted: January 16, 2017

Published: January 19, 2017

Copyright (c) 2017 by authors and Scientific Research Publishing Inc. This work is licensed under the Creative Commons Attribution International License (CC BY 4.0).

http://creativecommons.org/licenses/by/4.0/

\section{cc) (i) Open Access}

\begin{abstract}
Encryption and decryption method of three-dimensional objects uses holograms computer-generated and suggests encoding stage. Information obtained amplitude and phase of a three-dimensional object using mathematically stage transforms overlap stored on a digital computer. Different threedimensional images restore and develop the system for the expansion of the three-dimensional scenes and camera movement parameters. This article talks about these kinds of digital image processing algorithms as the reconstruction of three-dimensional model of the scene. In the present state, many such algorithms need to be improved in this paper proposing one of the options to improve the accuracy of such reconstruction.
\end{abstract}

\section{Keywords}

Three-Dimensional Scenes Restore Factorization Method,

Triangulation, VRML Model, Hybrid Algorithm

\section{Introduction}

Digital photo is a configurable performance fitting with the soundtracks of computers in which the division of the image to the minute network of image consists of a set of integers, often referred to. Pixels picture elements, or pixels preparation. Digital photo consisting of many thousands or millions of these pixels apparently is having a picture and sound. Each pixel represents a value of 255) can be explained by studying how-0 (dark black) to 255 (bright white), over the Values (0) Handle computers with numbers as the computers [1].

Work directly in numerical binary system which is the most the effectiveness of the use of ranges is compatible with the capabilities of number 2. Numbers in 
the range ( 0 to 255) can be absorbed by 1 byte, which consists of eight binary digits, or bits. A value of 8-bit value can store 28 or 256 value [2].

It may be necessary because of the contrast in the landscape lighting engineering and supply, weather conditions, and the noise the sensor and response. Each of these varies depending on the sensor selected and the platform used for data and conditions during data acquisition. Also, it may be desirable to convert and calibration data to a known radiation units or units of reflectance (absolute) to facilitate the comparison of data [3].

\section{Context and Aims}

Visual and data security encryption and key issues in optical communication systems for data storage and transfer of data protection from unauthorized access compared to traditional security systems, optical and security essentially provides two types of benefits. First, the parallel data which are more suitable for image encryption and rapid transmission of information. Second, treatment provides optics has been exploring the many degrees of freedom that the light beam may be encrypted, such as amplitude, phase, wavelength and polarization types. Different from the visual information security, different applications of information security visual verification systems, based on the optical link have been reported. These systems are connected to the two functions: First, lock, and always within the correlator; and the other is the key, the system by the user in the verification stage. The materials used, such as light scattering medium to hide the image data for the first phase of data protection, the use of this technique to hide to protect data from unauthorized access. Digital imaging is used to $\log$ efficiency. A triple security dimensions that combine encryption with random phase tri-dimensional digital technology system has been proposed threedimensional images by computer. This system provides information encryption $3 \mathrm{D}$ visually [4] [5].

Three-dimensional scenes restore using digital images is in demand and intensively develops all over the world [1]. Let us detach three the most typical tasks from the whole their complex:

1) Earth relief reconstruction using aerospace images for the needs of mapping and industrial facilities and emergency monitoring.

2) Premises plan reconstruction using images received from digital cameras and cine cameras for the needs of architecture and industrial premises rearrangement and equipment.

3) Mobile robots' vision systems.

Nowadays there are already some examples of such systems successful construction, however in general the task is far from its final solution; and the part of such systems requires precise measurements and calibration which is not always convenient. For example, on board of Terra satellite [6] there are two devices which allow you to restore three-dimensional scenes: MISR (9 identical telescopes with spatial resolution of $250 \mathrm{~m}$ ) and ASTER (stereo system with spa- 
tial resolution of $15 \mathrm{~m})$. Let us note that in case of high resolution survey $(\mathrm{d}=1$ $10 \mathrm{~m}$ ) for typical orbital altitude $\mathrm{h}=1000 \mathrm{~km}$ it is necessary to know cameras orientation with accuracy of $\sim \mathrm{d} / \mathrm{h}=10^{-5}-10^{-6} \mathrm{rad}$., and satellite coordinates with an accuracy of order $\mathrm{d}$. Such accuracies are achievable in principle, but they lead to complicated and expensive system with considerable mass and volume characteristics. That is why approaches that allow restoring such parameters directly from video data are of big interest.

The majority of systems described in the literature are based upon any single physical principle. In the meantime different approaches mutually complement each other and have different "bad cases". In summary it seems appropriate to construct combined system using the combination of different algorithms of three-dimensional scenes restore with choice of algorithm optimal for certain scene and its different parts.

\section{Method}

As a foundation for the system there was chosen a group of algorithms based upon matrix factorization [2]. The following reasons became the ground for such choice:

1) The possibility of restore of scene form and cameras motions, orientation and focal distances.

2) The possibility of work with dynamic scenes.

3) The method high accuracy due to big survey bases.

4) Low computational complexity.

As a result of approaches application three-dimensional coordinates of some object points are obtained with high accuracy, and a surface in the form of net or mesh, cameras coordinates and orientation, and in the presence of near objectscamera focal distances may be constructed point wise [7] Algorithms structure opens possibilities for integration with other approaches and contiguous data in two ways.

For near objects initial distance estimation may be performed by methods of defocus or stereo [8], or antecedent data (GPS, gyroscopes, etc.) may be used, that gives information for approximation selection and accelerates repeatability [9]. Coarse division of scene into areas of different remoteness may be performed, and for such areas the best approximation may be chosen. Usage of stereo or defocus with integrated camera allows detaching speedy objects and facilitating their characteristic point tracking.

Constructed mesh is a good initial approximation with well-determined nodes. Surface may be adjusted by methods [10] until dense map of scene depths is achieved. Adjusting method is chosen on the basis of image texture analysis and the obtained distance from cameras to the object's adjusted area estimated as the distance from camera to the surface set by mesh. General structure of the system is presented in Figure 1, and blocks realized at the moment are marked with thick frame. The system is realized in $\mathrm{C}++$ language and does not depend on operating system. 


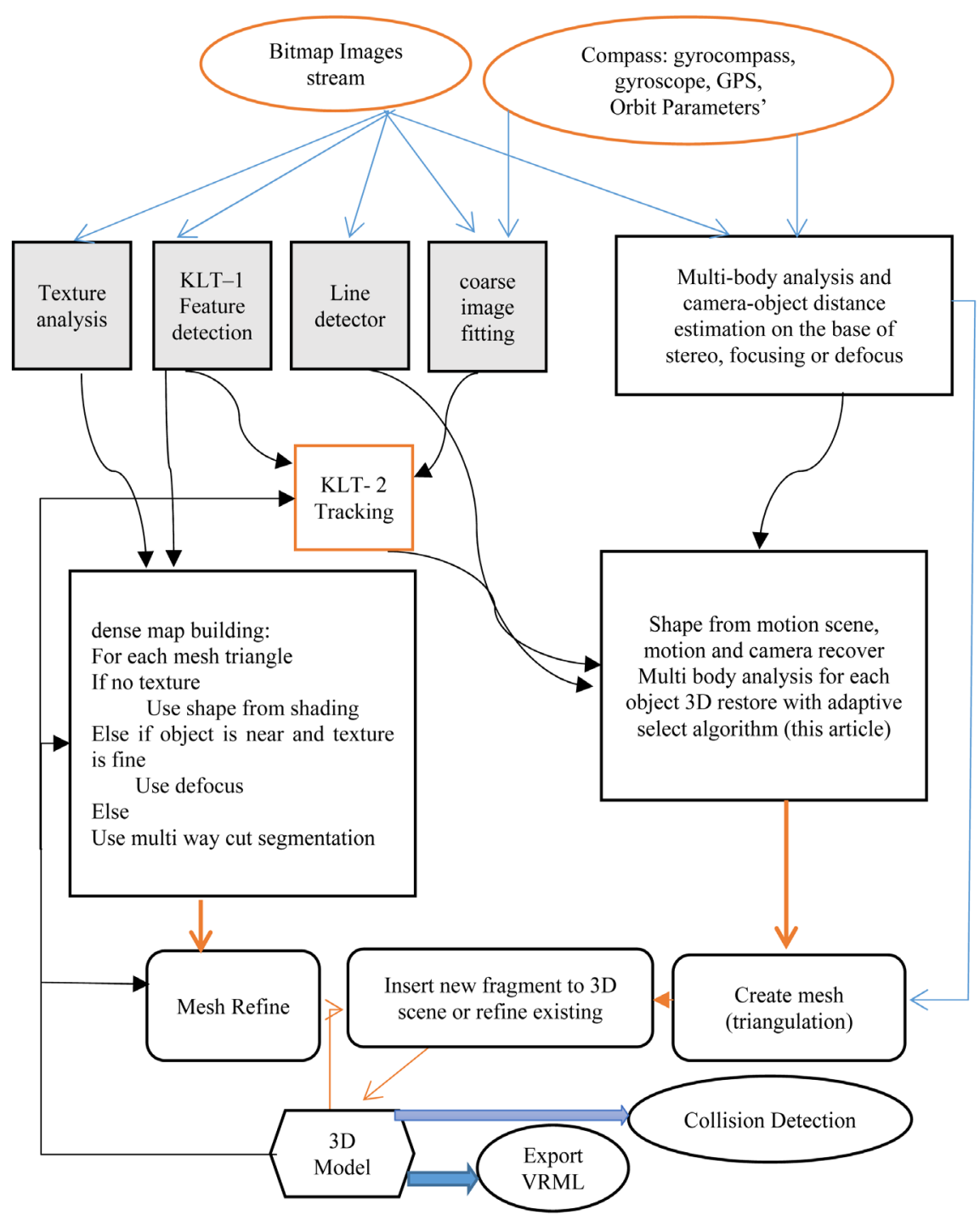

Figure 1. The flow chart of the system.

\section{Points Detection and Tracking}

The idea of tracking algorithm lies in the fact that at little differences of camera's position and orientation the area in later image may be obtained by means of displacement of certain area of the previous image. Then the displacement may be found from functional minimizing.

Where is displacement vector, is a window function. By means of brightness function linearization the task solution reduce itself to iterative solution of linear system [1]: Algorithm [1] works with gray images. It is suggested to generalize approach [1] in the event of multichannel images, and in particular of color ones. It may be easily achieved by brightness's in the functional to vectors whose components are corresponding to brightness's in corresponding channels. Then the final equation is of the same form as equation but and, where and are calculated independently from each channel as per formulas from [1].

Icon-based operation is carried out as per resolution pyramid. 


\section{Related Works}

When points tracking along the series of images it is possible both to loss points and add new ones. However for three-dimensional scene restore algorithm the availability of all tracked points on all frames is necessary. Consequently there appears a task of separation of the largest completely filled matrix where rows are frames, and columns are tracked points.

We offer to solve this task by searching for cliques of graph. Graph $g=\{X, E\}$ is constructed as follows. Its vertex set shall be all frames $\mathrm{F}$ and all tracked points P. Lines set $\mathrm{E}$ shall be set by two conditions. Subsets F and P are fully dependent sets. The line between vertexes and exists only in case if mark p presents in frame $f$ (see Figure 2).

When singling out from the graph of overlapping cliques there is a possibility to restore separate scene parts with their subsequent clipping due to common points. This allows restoring the scene more completely because the algorithms require all tracked points to be seen on the frame that is by no means always practicable. To find the clique the [11] algorithm is used.

When singling out from the graph of overlapping cliques there is a possibility to restore separate scene parts with their subsequent clipping due to common points. This allows to restore the scene more completely because the algorithms [2] [3] [4] require all tracked points to be seen on the frame that is by no means always practicable. To find the clique the [12] algorithm is used.

\section{Three-Dimensional Scene Restore Algorithm}

Serial algorithms and iteration algorithm in geometrical projection are realized in the system. Let us point out that the algorithm on each iteration step uses scaled orthography approximation that theoretically allows breaking iteration loop and confining you to approximate solution. Since the iteration algorithm extracts additional data from perspective distortions, then in case of longer distance to the object iterations do not lead to higher accuracy. The issue of iteration shutdown criterion comes under review in section devoted to results.

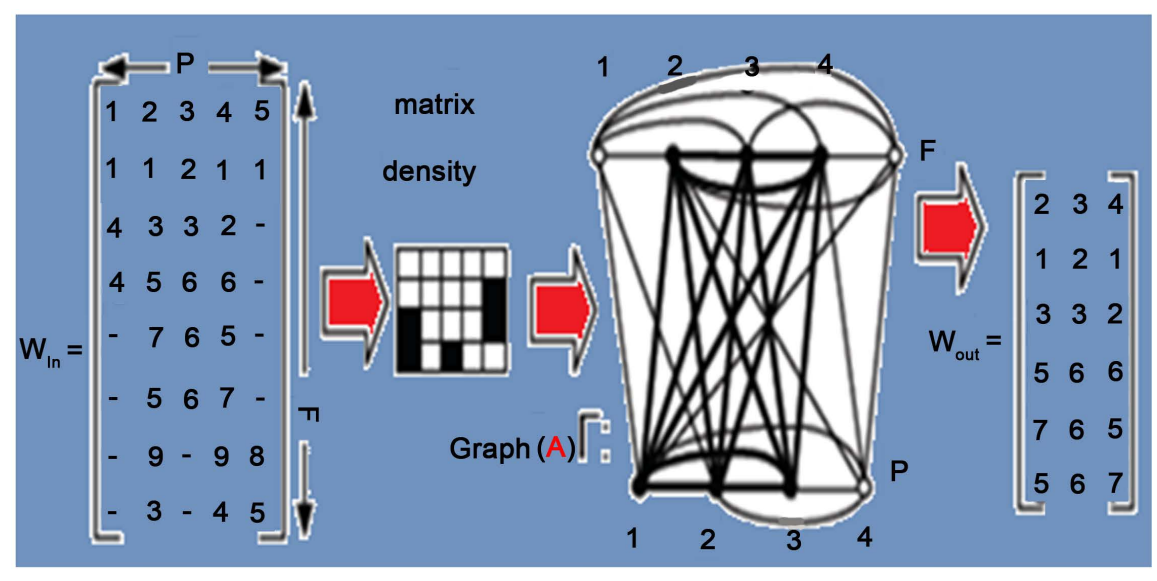

Figure 2. Search for dense sub matrix through clique of graph A, where F, P is a number of frames and points (marks). 


\section{Three-Dimensional Scene Construction in the Form of Mesh, Export to VRML}

The result of restore task solution is coordinates and cameras orientation, focal distances (in case of iteration algorithm usage) and three-dimensional coordinates of those object points that were detected and successfully tracked. Now we need to construct the object surface in the form of mesh due to these points. In reality the following approach is used. Since on every frame projection mutual arrangement of marks is always correct, and for the frame under number $f$ two-dimensional coordinates of marks on this stage are available and are contained in matrix rows $2 \mathrm{f}+1$ and $2 \mathrm{f}+2(3)$, then the triangulation is performed in frame's plane in two-dimensional space as per these points. Then obtained connections are formally applied to restore three-dimensional coordinates of corresponding points. Such approach allows to apply quick algorithm and excludes the possibility of line joining of points from different surfaces (sides) of the object, e.g. during the object circular survey, because such points cannot be seen on the image simultaneously. The restored surface coordinates and camera directions may be exported into VRML format with strained textures 4-Related works.

\section{Analysis}

Provided algorithm tested on real and synthetic data. For synthetic data in the form of trihedral angle diagrams of comparative restoration accuracy in different approximations depending on ratio of object distance $L$ to object size and are provided.

In Figure 3 and Figure 4, it can be seen that at short distances the method accuracy essentially exceeds the other ones. However with increase of distance different approximations accuracy becomes almost equal, and beginning with certain distance accuracy for method becomes comparable with or worse than for linear methods, It is connected with disappearance of perspective distortions in digital images. That is why the system must automatically switch to approximation of scaled orthography or Para perspective method.

Criterion for switch in the system was chosen as per results of numerical modeling on synthetic data described above. Dimensionless parameter L/a has been taken for the basic analysis parameter. It is evident that the accuracy of restoration depends on other parameters as well:

- Camera angular resolution;

- Restorable object angular dimension;

- Maximum camera motion ratio to average object distance;

- Angle of camera's rounding the object.

Numerical modeling results show that there is a small dependence of threshold value from these parameters, see In Figure 3 and Figure 4 real scenes restore is presented.

That reflects the proportion of documents retrieved from the site that are models. We maintain a Hash table of visited URLs to avoid visiting pages more 


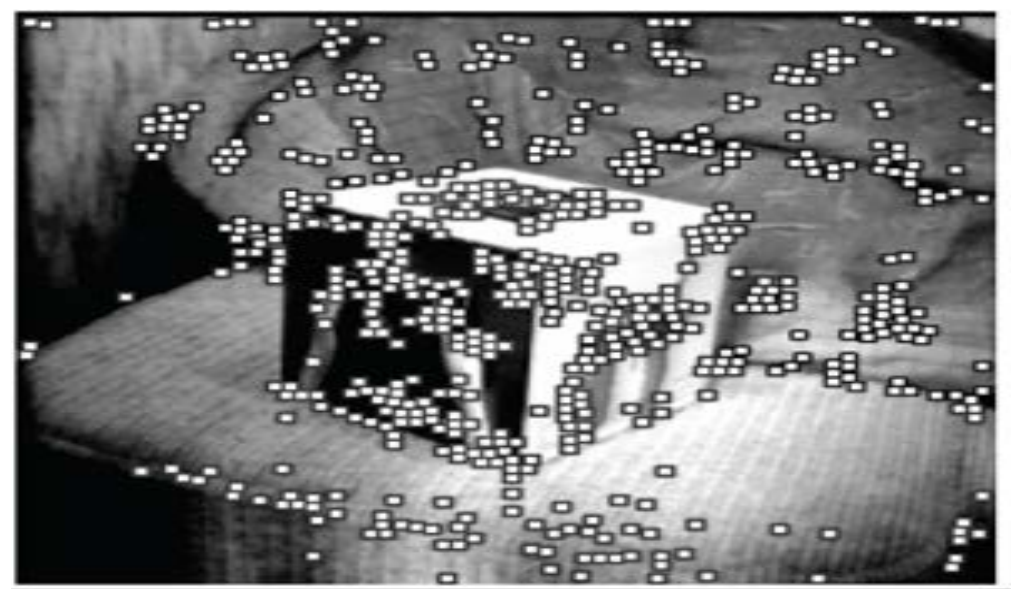

Figure 3. Frame with highlighted characteristic points.

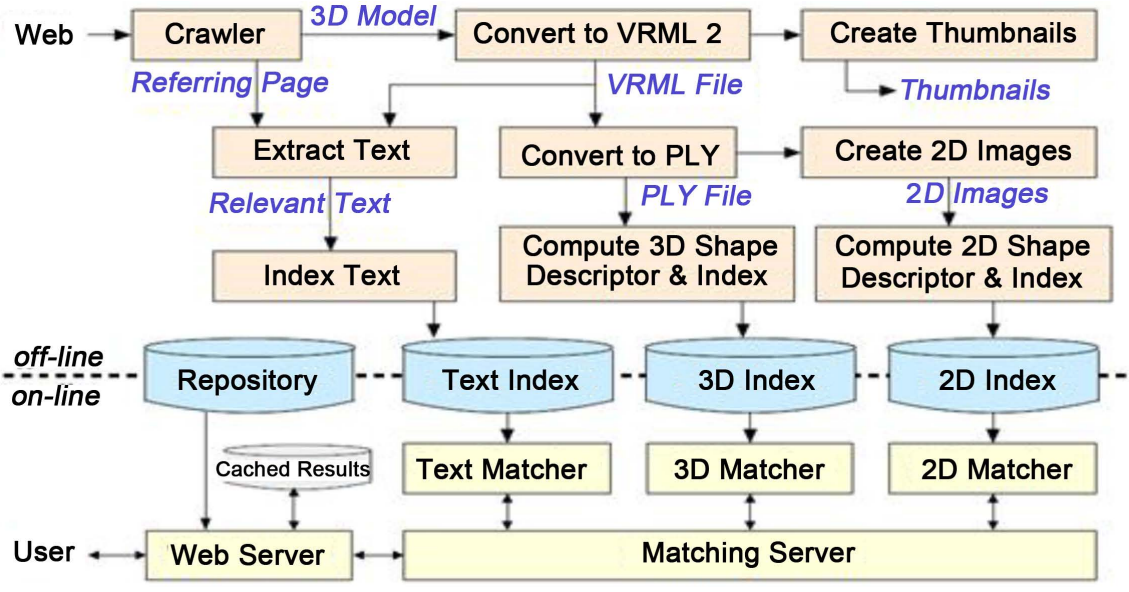

Figure 4. Flow of data through the search engine.

than once. Every downloaded 3D model goes through several preprocessing steps. We convert first to the VRML.

Our spherical harmonics method also can be indexed effectively. Figure 4 shows the average time (in seconds) required to find the 16 closest matches in databases of increasing size. Note that the search time grows sub linearly, and the total search time for a database of 17,500 models is less than 0.25 seconds.

\section{Conclusions}

Method is used to encrypt a 3D scene using random phase mask. Single and multiple phase masks are used as per sequence of digital images are developed. By means of numerical modeling, the criterion of computing (approximation) model adaptive selection was found. Restore accurate estimation was carried out. A new approach to dense measurements submatrix separation was suggested.

The approach based upon searching for clique of graph. It was shown that as applied to aerospace systems, high accuracy of relief restore and camera orientation restore accuracy are accessible. It is determined that the weakest point of the system's current condition is the requirement for small measurement of images 
between pairs of sequenced frames, and planned block of coarse integration is essential. In case of large distances, alternative way of forming disambiguation is necessary, that is based for example upon coarse knowledge of camera displacement between some pairs of frames.

\section{Acknowledgements}

This work was supported by the Deanship of Scientific Research, Jerash University. The author Prof. Al-Khawaldeh Igried is thankful to the AL-Balqa Applied University for their support and author Al smadi T. A to Jerash University by using their Laboratories and premises. We thank to reviewers for their valuable suggestions to enhance the quality of our article. This work has been carried out during sabbatical leave granted to the author Prof. Al-Khawaldeh Igried from AL-Balqa Applied University (BAU) during the academic year 2015/2016.

\section{References}

[1] Al Smadi, T.A. and Maitah, M. (2013) An Efficiency and Algorithm Detection for Stenography in Digital Symbols. International Journal of Computer Network and Information Security, 6, 34.

[2] Nishchal, N.K. and Naughton, T.J. (2011) Flexible Optical Encryption with Multiple Users and Multiple Security Levels. Optics Communications, 284, 735-739. https://doi.org/10.1016/j.optcom.2010.09.065

[3] Cho, M. and Javidi, B. (2013) Three-Dimensional Photon Counting Double-Random-Phase Encryption. Optics Letters, 38, 3198-3201. https://doi.org/10.1364/OL.38.003198

[4] Al Smadi, T., et al. (2015) Artificial Intelligence for Speech Recognition Based on Neural Networks. Journal of Signal and Information Processing, 6, 66.

[5] Rosenfeld, A. and Kak, A.C. (2014) Digital Picture Processing. Elsevier.

[6] Marks, R. (2007) System and Method for Providing a Real-Time Three-Dimensional Interactive en Reinhardt, Tilman, and Robert Seidl. Method and Apparatus for Image Assisted Modeling of Three-Dimensional Scenes. US Patent No. 7193633.

[7] Cameron, C.D., Wilson, C.P. and De Braal, A. (Year) Three Dimensional Projection Display. US Patent Application No. 11/947717.

[8] Fischler, M.A. and Firschein, O., Eds. (2014) Readings in Computer Vision: Issues, Problem, Principles, and Paradigms. Morgan Kaufmann.

[9] Medalia, M. (2007) Method of Generating a Three-Dimensional Interactive Tour of a Geographic Location. US Patent Application No. 11/782979.

[10] Spooner, D.A., Chan, S. and Simmons, C.L. (2014) Methodology for 3D Scene Reconstruction from 2D Image Sequences. US Patent No. 8655052.

[11] Al Smadi, T. (2014) Real-Time Lane Detection for Driver Assistance System. Circuits and Systems, 2014.

[12] Momani, M.A., et al. (2011) GPS Ionospheric Total Electron Content and Scintillation Measurements during the October 2003 Magnetic Storm. American Journal of Engineering and Applied Sciences. 
Submit or recommend next manuscript to SCIRP and we will provide best service for you:

Accepting pre-submission inquiries through Email, Facebook, LinkedIn, Twitter, etc. A wide selection of journals (inclusive of 9 subjects, more than 200 journals)

Providing 24-hour high-quality service

User-friendly online submission system

Fair and swift peer-review system

Efficient typesetting and proofreading procedure

Display of the result of downloads and visits, as well as the number of cited articles Maximum dissemination of your research work

Submit your manuscript at: http://papersubmission.scirp.org/

Or contact jsip@scirp.org 